\title{
Evaluation of NHS Grampian's child healthy weight programmes: Engagement and impact
}

\author{
F. Douglas ${ }^{1}$, L. C. A. Craig ${ }^{1}$, J. Clark ${ }^{1}$, T. Griffin ${ }^{1}$, C. Comerford ${ }^{2}$ \\ ${ }^{1}$ Public Health Nutrition Research Group, University of Aberdeen, Aberdeen AB25 2ZD, UK and ${ }^{2}$ NHS Grampian, \\ Aberdeen $A B 15$ 6RE
}

Halting rising levels of overweight and obesity in children is a key priority for the Scottish Government. In December 2007, the "health improvement, efficiency, access and treatment" (HEAT) target (H3) to "achieve agreed completion rates for child healthy weight intervention programmes by 2010/11" was introduced ${ }^{(1)}$. NHS Boards across Scotland were tasked with the delivery of child healthy weight interventions to $13.5 \%$ of 5-15 year olds identified as overweight $\left(91^{\text {st }}-99.6^{\text {th }}\right.$ centile of UK 1990 BMI charts). Following the guidance for approved H3 targeted intervention programmes, NHS Grampian developed Eat, Play and Grow Well (EPGW) and Moray Community Health and Social Care Partnership (CHSCP) developed CHANGE 3.

The aim of this study was to evaluate these programmes to explore the engagement of parents and children focusing on (1) the referral and recruitment processes, and (2) experience and impacts of the programme amongst children, parents and health professionals.

Eligible participants included parents and children who had recently participated in EPGW or CHANGE 3 in the Grampian region of Scotland, parents who had chosen not to take part following their child's referral, and staff involved with delivery of the programmes. Face-to-face qualitative interviews took place at home with 10 participating parents and 12 children. Telephone interviews took place with 5 non-participating parents, and 14 staff. Using Framework Analysis ${ }^{(2)}$ that incorporated analytic techniques used within Grounded Theory $^{(3)}$, we identified key emergent themes and concepts.

Participating parents and children were generally positive about the programmes, but also identified a range of modifications that they felt would improve effectiveness and encourage more families to engage. The personal characteristics and attributes of staff delivering the programme emerged as crucial factors regarding engagement and satisfaction with the programme.

The main barrier to engagement identified by parents and health professionals was associated with notions of parents failing to acknowledge or accept that their children had been assessed as overweight. This view was particularly evident amongst parents whose child's BMI was just above the cut-off point for overweight. There was also some evident confusion amongst parents about children's BMI classification. Some parents also believed there was a general lack of knowledge about the programmes within their school community. Both issues might explain the lack of engagement amongst some families with overweight children.

Some staff were anxious about raising the issue with parents, while other more experienced staff, who believed they had good existing relationships with the families concerned, were less anxious about it. It was not possible to ascertain whether dietary or physical activity changes claimed by parents or children, as a result of the programmes, had actually occurred. Moreover, many staff members expressed doubt that families would be able to sustain any changes in the longer term without further support from a professional.

Given current concern over childhood weight trends, this research provides insights into the experiences and views of parents, children and health professionals involved with child healthy weight programmes, and identified possible improvements for existing programmes in Grampian. However, it also suggests that the problem of childhood obesity is not perceived by many parents in the same way as policy makers or health professionals, and further research is required to understand their perspectives in more depth.

Funded by NHS Grampian

1. The Scottish Government (2007) Better Health, Better Care: action plan. http://www.scotland.gov.uk/About/scotPerforms/indicators/childrensBMI

2. Ritchie J \& Lewis J (2003) Qualitative Research Practice. Sage Publications.

3. Glaser BG \& Strauss AL (1967) Discovery of Grounded Theory. Strategies for Qualitative Research. Sociology Press. 